\title{
Mucoepidermoid carcinoma of the trachea
}

\author{
Moh'd Al-Halawani ${ }^{1}$, Yazan Abdeen ${ }^{2}$ \\ ${ }^{1}$ Division of Pulmonary and Critical Care Medicine, SUNY Downstate Medical Center, Brooklyn, NY 11203, USA; ${ }^{2}$ Pulmonary Department, Mercy \\ Clinic, Fort Smith, AR 72903, USA
}

Correspondence to: Moh'd Al-Halawani, MD. Division of Pulmonary and Critical Care Medicine, SUNY Downstate Medical Center, Brooklyn, NY 11203, USA. Email: mhalawanimd@gmail.com.

Submitted Apr 17, 2017. Accepted for publication May 13, 2017.

doi: 10.21037/qims.2017.06.01

View this article at: http://dx.doi.org/10.21037/qims.2017.06.01

A 24-year-old gentleman, previously medically free, presented with symptoms of cough and intermittent hemoptysis for several weeks. Laboratory work up was within normal limits. CT-scan of the neck and chest was done and revealed a $1.5 \times 1.5 \times 0.9 \mathrm{~cm}$ lobulated intraluminal tracheal mass arising from the posterior tracheal wall at the level of upper manubrium, with possible invasion of mediastinal fat posterior to the tracheal wall. There was no evidence of lymphadenopathy. Flexible bronchoscopy showed a large tracheal tumor (Figure 1) obstructing $60-70 \%$ of the trachea. Needle biopsy revealed low-grade mucoepidermoid carcinoma (Figure 2).

The patient underwent open partial tracheal resection and reconstruction under rigid bronchoscopy guidance. No further therapy was provided, and he remained asymptomatic on subsequent follow up. A 2-year post resection surveillance bronchoscopy of the surgical site is shown (Figure 3).

Primary salivary-gland tumors of the lung are extremely rare, comprising only $0.1-0.2 \%$ of primary lung cancers and less than $1 \%$ of primary malignant bronchial tumors. Mucoepidermoid carcinoma (MEC) is a salivary-gland type of lung cancer that originates from the submucosal glands of the tracheobronchial tree. It affects patients of age 30 years and older $(1,2)$.

Patients may present with symptoms of bronchial irritation including cough, hemoptysis, wheezing and post-

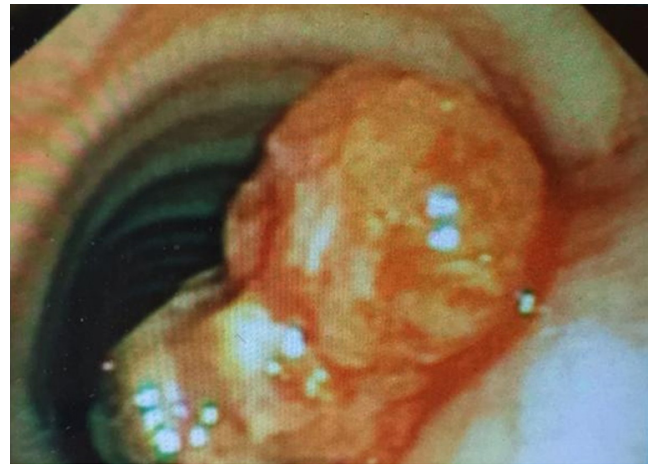

Figure 1 Endotracheal tumor visualised during bronchoscopy.

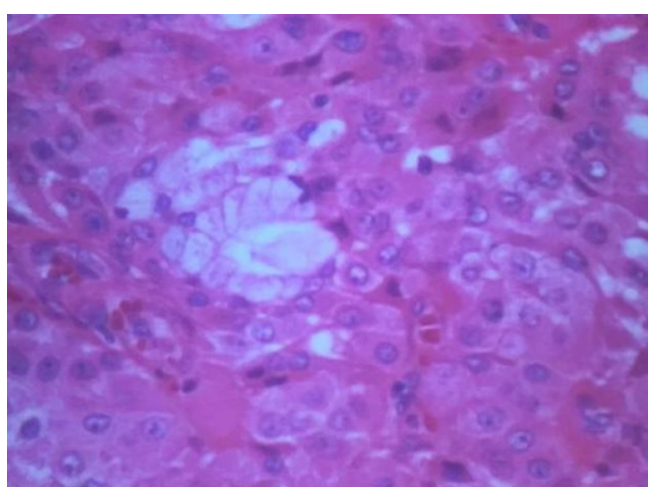

Figure 2 High-power field microscopy showing epithelial cells with prominent nuclei and mucin accumulation in the cytoplasm, consistent with mucoepidermoid carcinoma. 


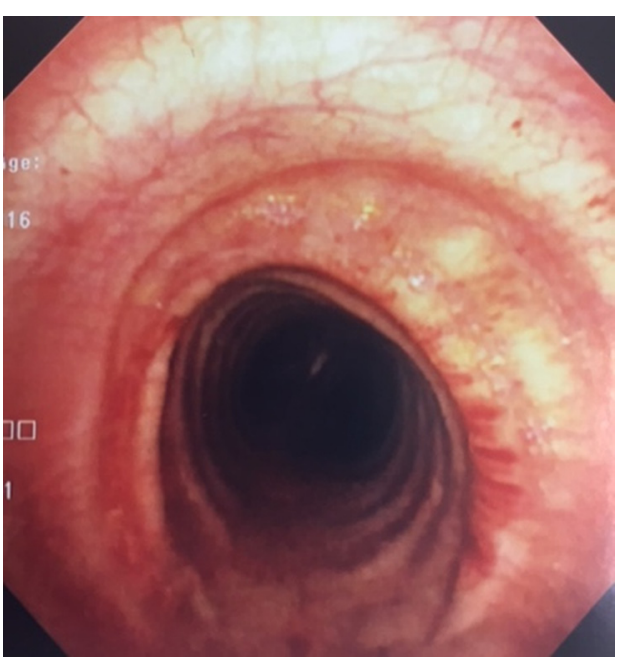

Figure 3 Two years post-resection surveillance bronchoscopy showing no recurrence of the tumor.

obstructive pneumonia, but may also be asymptomatic $(1,2)$.

MEC is classified as low-grade or high-grade based on histologic appearance.

Surgical resection is the mainstay of treatment, and usually carries good prognosis. Low-grade malignant tumors of the

Cite this article as: Al-Halawani M, Abdeen Y. Mucoepidermoid carcinoma of the trachea. Quant Imaging Med Surg 2018;8(2):259-260. doi: 10.21037/qims.2017.06.01 bronchial airway are often managed with open resection. High-grade MEC carries a higher risk of distant metastasis and worse prognosis even with surgery $(2,3)$.

\section{Acknowledgements}

None

\section{Footnote}

Conflicts of Interest: The authors have no conflicts of interest to declare.

\section{References}

1. Abu Saleh WK, Aljabbari O, Ramchandani M. Mucoepidermoid Carcinoma of the Tracheobronchial Tree. Methodist Debakey Cardiovasc J 2015;11:192-4.

2. Shen C, Che G. Clinicopathological analysis of pulmonary mucoepidermoid carcinoma. World J Surg Oncol 2014;12:33.

3. Kawano O, Yuki D, Fukai I, et al. Successful treatment of mucoepidermoid carcinoma in the left main bronchus. Surg Case Rep 2015;1:85. 